\title{
Analysis of demersal assemblages off the Tuscany and Latium coasts (north-western Mediterranean)*
}

\author{
FRANCO BIAGI $^{1}$, PAOLO SARTOR ${ }^{2}$, GIAN DOMENICO ARDIZZONE ${ }^{3}$, \\ PAOLA BELCARI ${ }^{1}$, ANDREA BELLUSCIO ${ }^{3}$ and FABRIZIO SERENA ${ }^{4}$ \\ ${ }^{1}$ Dipartimento di Scienze dell'Uomo e dell'Ambiente, Università di Pisa, Via Volta 6, 56126 Pisa, Italy. \\ E-mail: biagifra@discat.unipi.it \\ ${ }^{2}$ Centro Interuniversitario di Biologia Marina ed Ecologia Applicata, Livorno, Italy. \\ ${ }^{3}$ Dipartimento di Biologia Animale e dell'Uomo, Università di Roma "La Sapienza", Roma, Italy. \\ ${ }_{4}^{4}$ Agenzia Regionale per l'Ambiente Toscana, Livorno, Italy.
}

\begin{abstract}
SUMMARY: A four-year time series (1994-1997) of groundfish trawl surveys performed within the European Union Project "MEDITS" (Mediterranean International Trawl Surveys), was analysed to identify and describe the fish assemblages along the continental shelf and slope of Tuscany and Latium (Italy), in the north-western Mediterranean. Cluster analysis was used to group samples with similar species composition in terms of abundance, biomass and frequency of occurrence. Results allowed the identification of four to five broad assemblages along the depth gradient: a strictly coastal group $(<50$ $\mathrm{m}$ depth), two groups in the upper and lower part of the continental shelf (essentially 50-200 m), an epibathyal group (200$450 \mathrm{~m}$ ) and a group derived from hauls made at depths greater than $450 \mathrm{~m}$. Each assemblage corresponded to a faunistic association with relatively homogeneous and persistent species composition, biomass and density indices.
\end{abstract}

Key words: assemblages, cluster analysis, demersal resources, western Mediterranean

\section{INTRODUCTION}

The fauna of the Mediterranean basin is characterised by a high biodiversity, i.e. by a high number of species, some endemic to the area, some with a wider geographical distribution and others recently immigrating into the Mediterranean waters (Maurin, 1962; Tortonese, 1987; Fredj et al., 1992). This aspect is mainly due to the geological evolution of the basin and to its ecological and climatic features. The existence of such biological diversity certainly contributes to the health of the Mediterranean fisheries.

\footnotetext{
*Received Novemver 7, 2000. Accepted February 14, 2002.
}

Classical stock assessment tools indicate in most cases a general state of overexploitation and overcapacity of the fishing fleets (Caddy and Sharp, 1986). However, monospecific approaches are usually the rule. In the last 15 years there has been a growing perception that it is often ineffective to base fisheries management policies exclusively on single species approaches. High levels of variability in productivity, in abundance, in size and species composition, are not predictable from single species models. However, problems can arise when choosing management options in a multispecies fishery context (Caddy and Sharp, 1986; Brugge and Holden, 1991).

A more holistic approach to resource management is evidently needed. In practice, management 
should be based on ecosystem principles: to maintain the full ensemble of species while sustaining the harvest of desired species (Tyler et al., 1982; Tyler, 1988). Overexploitation alters not only the size of stocks but also the ecosystems through the successive removal of large long-lived species and largesized specimens to the point where trophic linkages, biodiversity and resilience have all been lowered (Caddy and Sharp, 1986).

The aim of this work is to analyse the structure of demersal assemblages (finfish, crustaceans and cephalopods), on a temporal and spatial scale and to specify the set of species characterising each group and those species responsible for sample groupings. From this perspective, data arising from "MEDITS" trawl surveys carried out in the years 1994-1997 along the Tuscany and Latium coasts, were analysed. Some previous information about fish assemblages in this area has been analysed by Biagi et al. (1989) and Abella and Serena (1995a, b).

\section{MATERIAL AND METHODS}

This study was carried out within the framework of the Mediterranean International Trawl Surveys Programme ("MEDITS") (Bertrand et al., 2000, 2002). Four experimental bottom trawl surveys were carried out in the years 1994-1997, in the springsummer period (essentially from June to July). The investigated area encompassed $36,536 \mathrm{~km}^{2}$, comprising the Italian coasts along Tuscany and Latium (Ligurian and Tyrrhenian seas). This area was subdivided into three sub-areas: from Magra River to Elba Island (sub-area 1), from Elba to Giannutri Island (sub-area 2) and from Giannutri to Garigliano River (sub-area 3) (Fig. 1).

In the first survey, each sub-area was fully covered on a random stratified sampling basis according to the following depth strata: stratum A: $10-50 \mathrm{~m}$; stratum B: $50-100 \mathrm{~m}$; stratum C: $100-200 \mathrm{~m}$; stratum D: $200-500 \mathrm{~m}$; stratum E: $500-800 \mathrm{~m}$. The allocation of hauls was proportional to the surface of each

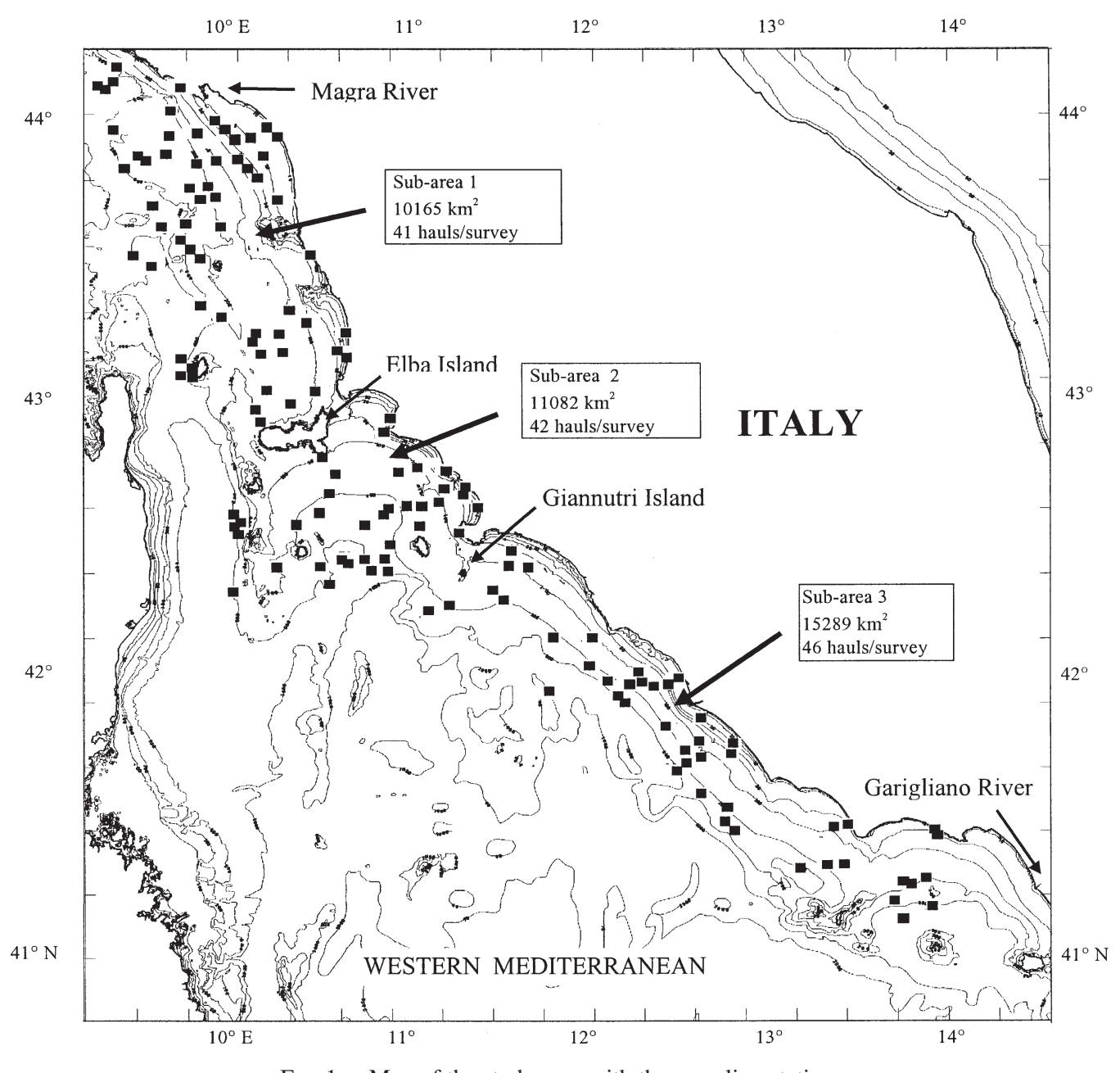

FIG. 1. - Map of the study area with the sampling stations. 
bathymetric stratum. The same hauls were replicated in successive surveys. Globally, 129 hauls were made in day time hours in each survey, from 12 to $751 \mathrm{~m}$ of mean depth, distributed as follows according to the different depth strata: 18 hauls in stratum A, 18 in stratum B, 36 in stratum C, 33 in stratum D, and 24 in stratum E (Fig. 1).

Haul duration was 30 minutes at depths shallower than $200 \mathrm{~m}$ and one hour at depths greater than $200 \mathrm{~m}$. All the surveys were carried out with the same otter bottom trawler and the same type of trawl net, having a cod end with stretched mesh sizes of $10 \mathrm{~mm}$ (knot to knot). Further information on the sampling design and on the characteristics of the gear is available in Bertrand et al. (2000, 2002).

The species composition of the different taxocoenosis (finfish, crustaceans and cephalopods) was studied by analysing abundance and biomass data coming from each survey. Raw catch data (biomass and number of specimens collected) of each haul were converted into abundance (number of specimens $\left./ \mathrm{km}^{2}\right)$ and biomass indices $\left(\mathrm{kg} / \mathrm{km}^{2}\right)$, utilising a specifically developed software (Souplet, 1996). In order to identify faunistic associations, cluster analysis was applied to the abundance and biomass species-stations matrices of each survey; occasional species (those caught only once time in each year) were not considered in this analysis. In addition, the strictly pelagic species (such as anchovy, Engraulis encrasicolus, and sardine, Sardina pilchardus) were eliminated from the analysis because, in most cases, they were assumed to be captured when the trawl gear was not sampling at the bottom; this could lead to some distortion in grouping the sampling into different assemblages.

Because the survey in 1994 was mostly aimed at standardising and calibrating the sampling gear, cluster analysis was performed using the data sets of the years 1995-1997. To reduce variability and skewness, data transformations were applied: double square root for the abundance data and $\log (x+1)$ for the biomass data.

Similarity levels between the stations were calculated through the Bray-Curtis index (Clifford and Stephenson, 1975). A group average fusion strategy, UPGMA, was used as clustering algorithm (Sneath and Sokal, 1973). Species composition of each assemblage was described in terms of percentage contribution of each species at the similarity level of each group. The above mentioned analyses were computed with the PRIMER statistical package (rel. 4.0) (Clarke and Warwick, 1994).

\section{RESULTS}

During the four surveys, a total of 253 species (32 Cephalopoda, 47 Crustacea, 21 Condrichthyes and 153 Osteichthyes) were collected (Table 1). Out of these, 165 species $(65.6 \%)$ occurred in all the three sub-areas $(71.0 \%, 68.1 \%, 52.4 \%$ and $63.8 \%$, respectively for cephalopods, crustaceans, cartilaginous and bony fishes). A total of 194 species (12 exclusive) was collected in sub-area 1, 212 in subarea 2 (15 exclusive) and 209 in sub-area 3 (9 exclusive).

The number of species caught in each haul ranged from a minimum of 10 to a maximum of 44 . However, no evident bathymetric trend in number of species by haul was observed. Over the four years, the mean number of species per haul ranged between 20 and 40 .

Four major clusters of stations recurrently appeared in the dendrograms calculated for each survey (Figs. 2 and 3). Each cluster was closely associated with bottom depths. Actually, it was possible to identify a group composed of strictly coastal hauls down to $50 \mathrm{~m}$ depth, another group derived from most of the hauls carried out on the continental shelf (essentially between 60 and $220 \mathrm{~m}$ depth), a third cluster based on the hauls made along the upper part of the continental slope (from 200 to 450 $\mathrm{m}$ depth) and the last group from the deepest hauls (mostly deeper than 450-500 m). Regarding the continental shelf group, a further subdivision into two sub-groups could be done: a shallower group, with most hauls taken at depths of less than $130 \mathrm{~m}$, and another with the majority of hauls made at greater depths.

Cluster structure (regarding both biomass and abundance) remained essentially the same in the three years: about $90 \%$ of hauls was assigned to the same clusters over the studied period when four groups were retained, about $85 \%$ when five groups were considered (Table 2).

Although many species occurred in more than one group, each faunistic association was characterised by its own extent of species composition and catch rates (abundance and biomass) which distinguished them from each other. Moreover, these assemblages remained substantially unchanged over the study period for both data sets (abundance and biomass) (Table 3).

The coastal group was mostly characterised by Trachurus mediterraneus, Diplodus annularis, Alloteuthis spp., Pagellus erythrinus, Arnoglossus later- 
TABLE 1. -- List of the species collected during the surveys carried out in the period 1994-1997 off the Tuscany and Latium coasts. Cephalopod species names follow Mangold and Boletzky (1987), crustaceans follow Zariquiey Alvarez (1968) and fishes follow Whitehead et al. $(1984,1986)$

\begin{tabular}{|c|c|c|c|}
\hline CEPHALOPODA & Fam. Pandalidae & Fam. Myliobatidae & Gaidropsarus mediterraneus \\
\hline Fam. Sepiidae & Chlorotocus crassicornis & Myliobatis aquila & Micromesistius poutassou \\
\hline Sepia elegans & Plesionika acanthonotus & Fam. Chimaeridae & Molva dipterygia \\
\hline Sepia officinalis & Plesionika antigai & Chimaera monstrosa & Molva molva \\
\hline Sepia orbignyana & Plesionika edwardsii & & Phycis blennoides \\
\hline Fam. Sepiolidae & Plesionika gigliolii & OSTEICHTHYES & Phycis phycis \\
\hline Rossia macrosoma & Plesionika heterocarpus & Fam. Clupeidae & Trisopterus minutus capelanus \\
\hline Neorossia caroli & Plesionika martia & Alosa fallax & Fam. Moridae \\
\hline Heteroteuthis dispar & Fam. Crangonidae & Sardina pilchardus & Gadella maraldi \\
\hline Stoloteuthis leucoptera & Philocheras echinulatus & Sardinella aurita & Lepidion lepidion \\
\hline Rondeletiola minor & Pontocaris cataphractus & Fam. Engraulidae & Mora moro \\
\hline Sepietta oweniana & Pontocaris lacazei & Engraulis encrasicolus & Fam. Trachichthyidae \\
\hline Sepiola intermedia & Pontophilus spinosus & Fam. Gonostomatidae & Hoplostethus mediterraneus \\
\hline Sepiola ligulata & Fam. Nephropidae & Gonostoma denudatum & Fam. Zeidae \\
\hline Fam. Loliginidae & Nephrops norvegicus & Fam. Sternoptychidae & Zeus faber \\
\hline Alloteuthis media & Fam. Polychelidae & Argyropelecus hemigymnus & Fam. Caproidae \\
\hline Alloteuthis subulata & Polycheles typhlops & Maurolicus muelleri & Capros aper \\
\hline Loligo forbesi & Fam. Axiidae & Fam. Photichthyidae & Fam. Serranidae \\
\hline Loligo vulgaris & Calocaris macandreae & Ichthyococcus ovatus & Polyprion americanus \\
\hline Fam. Ctenopterygidae & Fam. Palinuridae & Fam. Chauliodontidae & Serranus cabrilla \\
\hline Ctenopterix sicula & Palinurus elephas & Chauliodus sloani & Serranus hepatus \\
\hline Fam. Enoploteuthidae & Palinurus mauritanicus & Fam. Stomiidae & Fam. Moronidae \\
\hline Abralia verany & Fam. Paguridae & Stomias boa & Dicentrarchus labrax \\
\hline Fam. Octopoteuthidae & Pagurus alatus & Fam. Argentinidae & Fam. Apogonidae \\
\hline Octopoteuthis sicula & Pagurus prideaux & Argentina sphyraena & Epigonus constanciae \\
\hline Fam. Histioteuthidae & Fam. Galateidae & Glossanodon leioglossus & Epigonus denticulatus \\
\hline Histioteuthis bonnellii & Munida intermedia & Fam. Synodontidae & Epigonus telescopus \\
\hline Histioteuthis reversa & Munida tenuimana & Synodus saurus & Fam. Cepolidae \\
\hline Fam. Ommastrephidae & Fam. Dorippidae & Fam. Chlorophthalmidae & Cepola rubescens \\
\hline Illex coindetii & Medorippe lanata & Chlorophthalmus agassizii & Fam. Pomatomidae \\
\hline Todarodes sagittatus & Fam. Geryonidae & Fam. Myctophidae & Pomatomus saltator \\
\hline Todaropsis eblanae & Geryon longipes & Benthosema glaciale & Fam. Carangidae \\
\hline Fam. Onychoteuthidae & Fam. Homolidae & Ceratoscopelus maderensis & Trachurus mediterraneus \\
\hline Ancistroteuthis lichtensteinii & Paromola cuvieri & Diaphus metopoclampus & Trachurus picturatus \\
\hline Fam. Octopodidae & Fam. Portunidae & Diaphus rafinesquei & Trachurus trachurus \\
\hline Bathypolypus sponsalis & Bathynectes maravigna & Hygophum benoiti & Fam. Sciaenidae \\
\hline Eledone cirrhosa & Liocarcinus depurator & Hygophum hygomii & Umbrina cirrosa \\
\hline Eledone moschata & Liocarcinus vernalis & Lampanyctus crocodilus & Fam. Mullidae \\
\hline Octopus salutii & Macropipus tuberculatus & Lobianchia dofleini & Mullus barbatus \\
\hline Octopus vulgaris & Fam. Goneplacidae & Myctophum punctatum & Mullus surmuletus \\
\hline Pteroctopus tetracirrhus & Goneplax rhomboides & Notoscopelus elongatus & Fam. Sparidae \\
\hline Scaeurgus unicirrhus & Fam. Majidae & Symbolophorus veranyi & Boops boops \\
\hline Fam. Ocythoidae & Macropodia longipes & Fam. Paralepididae & Dentex dentex \\
\hline Ocythoe tuberculata & Maja crispata & Notolepis rissoi & Diplodus annularis \\
\hline & Maja squinado & Paralepis coregonoides & Diplodus sargus \\
\hline CRUSTACEA & & Fam. Nemichthyidae & Diplodus vulgaris \\
\hline STOMATOPODA & CHONDRICHTHYES & Nemichthys scolopaceus & Lithognathus mormyrus \\
\hline Fam. Squillidae & Fam. Hexanchidae & Fam. Nettastomatidae & Oblada melanura \\
\hline Rissoides pallidus & Hexanchus griseus & Nettastoma melanurum & Pagellus acarne \\
\hline Squilla mantis & Fam. Scyliorhinidae & Fam. Congridae & Pagellus bogaraveo \\
\hline DECAPODA & Galeus melastomus & Conger conger & Pagellus erythrinus \\
\hline Fam. Solenoceridae & Scyliorhinus canicula & Gnathophis mystax & Pagrus pagrus \\
\hline Solenocera membranacea & Scyliorhinus stellaris & Fam. Ophichthidae & Sarpa salpa \\
\hline Fam. Aristeidae & Fam. Oxynotidae & Dalophis imberbis & Sparus aurata \\
\hline Aristaeomorpha foliacea & Oxynotus centrina & Echelus myrus & Spondyliosoma cantharus \\
\hline Aristeus antennatus & Fam. Squalidae & Ophisurus serpens & Fam. Centracanthidae \\
\hline Gennadas elegans & Centrophorus granulosus & Fam. Notacanthidae & Centracanthus cirrus \\
\hline Fam. Penaeidae & Dalatias licha & Notacanthus bonapartei & Spicara flexuosa \\
\hline Funchalia woodwardi & Etmopterus spinax & Fam. Macroramphosidae & Spicara maena \\
\hline Penaeus kerathurus & Squalus acanthias & Macroramphosus scolopax & Spicara smaris \\
\hline Parapenaeus longirostris & Squalus blainvillei & Fam. Syngnathidae & Fam. Labridae \\
\hline Fam. Sergestidae & Fam. Torpedinidae & Hippocampus guttulatus & Acantholabrus palloni \\
\hline Sergestes arcticus & Torpedo marmorata & Hippocampus hippocampus & Lappanella fasciata \\
\hline Sergestes robustus & Torpedo torpedo & Fam. Macrouridae & Fam. Trachinidae \\
\hline Fam. Pasiphaeidae & Fam. Rajidae & Coelorhynchus coelorhynchus & Trachinus draco \\
\hline Pasiphaea multidentata & Raja asterias & Hymenocephalus italicus & Trachinus radiatus \\
\hline Pasiphaea sivado & Raja batis & Nezumia sclerorhynchus & Fam. Uranoscopidae \\
\hline Fam. Alpheidae & Raja clavata & Trachyrhynchus trachyrhynchus & Uranoscopus scaber \\
\hline Alpheus glaber & Raja miraletus & Fam. Merlucciidae & Fam. Trichiuridae \\
\hline Fam. Hippolytidae & Raja montagui & Merluccius merluccius & Lepidopus caudatus \\
\hline Ligur ensiferus & Raja oxyrinchus & Fam. Gadidae & Fam. Scombridae \\
\hline Fam. Processidae & Fam. Dasyatidae & Antonogadus megalokynodon & Scomber japonicus \\
\hline Processa mediterranea & Dasyatis violacea & Gadiculus argenteus & Scomber scombrus \\
\hline
\end{tabular}


TABLE 1 (Cont.). -- List of the species collected during the surveys carried out in the period 1994-1997 off the Tuscany and Latium coasts.

\begin{tabular}{lccc}
\hline & & & \\
Fam. Gobiidae & Centrolophus niger & Trigla lyra & Microchirus variegatus \\
Aphia minuta & Fam. Sphyraenidae & Trigloporus lastoviza & Monochirus hispidus \\
Deltentosteus quadrimaculatus & Sphyraena sphyraena & Fam. Peristediidae & Solea impar \\
Gobius geniporus & Fam. Mugilidae & Peristedion cataphractum & Solea lascaris \\
Gobius niger & Liza ramada & Fam. Citharidae & Solea vulgaris \\
Lesueurigobius friesii & Liza saliens & Fam. Scoph linguatula & Fam. Cynoglossidae \\
Lesueurigobius suerii & Fam. Scorpaenidae & Lepidorhombus boscii & Symphurus ligulatus \\
Pomatoschistus marmoratus & Helicolenus dactylopterus & Lepidorhombus whiffiagonis & Fam. Balistidae \\
Fam. Callionymidae & Scorpaena elongata & Psetta maxima & Balistes carolinensis \\
Callionymus maculatus & Scorpaena notata & Scophthalmus rhombus & Fam. Molidae \\
Callionymus risso & Scorpaena porcus & Fam. Bothidae & Mola mola \\
Synchiropus phaeton & Scorpaena scrofa & Arnoglossus imperialis & Fam. Gobiesocidae \\
Fam. Blenniidae & Fam. Triglidae & Arnoglossus laterna & Diplecogaster bimaculata \\
Blennius ocellaris & Aspitrigla cuculus & Arnoglossus rueppelli & Fam. Lophiidae \\
Fam. Bythitidae & Aspitrigla obscura & Arnoglossus thori & Lophius budegassa \\
Bellottia apoda & Eutrigla gurnardus & Bothus podas & Lophius piscatorius \\
Fam. Ophidiidae & Lepidotrigla cavillone & Fam. Soleidae & \\
Benthocometes robustus & Lepidotrigla dieuzeidei & Buglossidium luteum & \\
Fam. Centrolophidae & Trigla lucerna & & \\
\hline
\end{tabular}

TABLE 2. - Characteristics of the clusters in terms of depth ranges and number of stations.

\begin{tabular}{|c|c|c|c|c|c|c|c|}
\hline \multirow[t]{2}{*}{ A) Considering 4 groups } & \multicolumn{3}{|c|}{ Biomass data } & & \multicolumn{3}{|c|}{ Abundance data } \\
\hline & 1995 & 1996 & 1997 & & 1995 & 1996 & 1997 \\
\hline $\begin{array}{l}\text { Group 1 } \\
\text { Depth range (m) } \\
\mathrm{N}^{\circ} \text { of stations } \\
\text { Stations always present }\end{array}$ & $\begin{array}{c}12-67 \\
19 \\
\text { roup: } 18\end{array}$ & $\begin{array}{c}12-47 \\
18 \\
4.7 \%)\end{array}$ & $\begin{array}{c}12-47 \\
18\end{array}$ & $\begin{array}{l}\text { Group } 1 \\
\text { Depth range (m) } \\
\mathrm{N}^{\circ} \text { of stations } \\
\text { Stations always p }\end{array}$ & $\begin{array}{c}12-67 \\
19 \\
\text { oup: } 18(9\end{array}$ & $\begin{array}{l}12-47 \\
18\end{array}$ & $\begin{array}{c}12-47 \\
18\end{array}$ \\
\hline $\begin{array}{l}\text { Group } 2 \\
\text { Depth range (m) } \\
\mathrm{N}^{\circ} \text { of stations } \\
\text { Stations always present }\end{array}$ & $\begin{array}{c}62-195 \\
52 \\
\text { roup: } 51\end{array}$ & $\begin{array}{c}62-275 \\
56 \\
1.0 \%)\end{array}$ & $\begin{array}{c}62-218 \\
56\end{array}$ & $\begin{array}{l}\text { Group 2 } \\
\text { Depth range (m) } \\
\mathrm{N}^{\circ} \text { of stations } \\
\text { Stations always p }\end{array}$ & $\begin{array}{c}62-275 \\
55 \\
\text { oup: } 52(9\end{array}$ & $\begin{array}{l}62-249 \\
56\end{array}$ & $\begin{array}{c}62-218 \\
56\end{array}$ \\
\hline $\begin{array}{l}\text { Group } 3 \\
\text { Depth range (m) } \\
\mathrm{N}^{\circ} \text { of stations } \\
\text { Stations always present }\end{array}$ & $\begin{array}{c}202-413 \\
27 \\
\text { roup: } 27\end{array}$ & $\begin{array}{c}202-428 \\
27 \\
7.1 \%)\end{array}$ & $\begin{array}{c}202-505 \\
31\end{array}$ & $\begin{array}{l}\text { Group } 3 \\
\text { Depth range (m) } \\
\mathrm{N}^{\circ} \text { of stations } \\
\text { Stations always p }\end{array}$ & $\begin{array}{c}202-413 \\
24 \\
\text { oup: } 24(7\end{array}$ & $\begin{array}{l}202-434 \\
27 \\
\%)\end{array}$ & $\begin{array}{c}202-505 \\
31\end{array}$ \\
\hline $\begin{array}{l}\text { Group } 4 \\
\text { Depth range (m) } \\
\mathrm{N}^{\circ} \text { of stations } \\
\text { Stations always present }\end{array}$ & $\begin{array}{c}393-751 \\
31 \\
\text { roup: } 24\end{array}$ & $\begin{array}{c}454-751 \\
28 \\
7.4 \%)\end{array}$ & $\begin{array}{c}513-751 \\
24\end{array}$ & $\begin{array}{l}\text { Group } 4 \\
\text { Depth range (m) } \\
\mathrm{N}^{\circ} \text { of stations } \\
\text { Stations always p }\end{array}$ & $\begin{array}{c}393-751 \\
31 \\
\text { oup: } 24(7\end{array}$ & $\begin{array}{l}454-751 \\
28 \\
\%)^{2}\end{array}$ & $\begin{array}{c}513-751 \\
24\end{array}$ \\
\hline B) Considering 5 groups & \multicolumn{3}{|c|}{ Biomass data } & & \multicolumn{3}{|c|}{ Abundance data } \\
\hline $\begin{array}{l}\text { Group 1 } \\
\text { Depth range (m) } \\
\mathrm{N}^{\circ} \text { of stations } \\
\text { Stations always present }\end{array}$ & $\begin{array}{c}12-67 \\
19 \\
\text { roup: } 18\end{array}$ & $\begin{array}{c}12-47 \\
18 \\
4.7 \%)\end{array}$ & $\begin{array}{c}12-47 \\
18\end{array}$ & $\begin{array}{l}\text { Group 1 } \\
\text { Depth range (m) } \\
\mathrm{N}^{\circ} \text { of stations } \\
\text { Stations always p }\end{array}$ & $\begin{array}{c}12-67 \\
19 \\
\text { oup: } 18\end{array}$ & $\begin{array}{l}12-47 \\
18\end{array}$ & $\begin{array}{c}12-47 \\
18\end{array}$ \\
\hline $\begin{array}{l}\text { Group 2a } \\
\text { Depth range (m) } \\
\mathrm{N}^{\circ} \text { of stations } \\
\text { Stations always present }\end{array}$ & $\begin{array}{c}62-131 \\
31 \\
\text { roup: } 27\end{array}$ & $\begin{array}{c}62-123 \\
30 \\
7.1 \%)\end{array}$ & $\begin{array}{c}62-131 \\
28\end{array}$ & $\begin{array}{l}\text { Group 2a } \\
\text { Depth range (m) } \\
\mathrm{N}^{\circ} \text { of stations } \\
\text { Stations always p }\end{array}$ & $\begin{array}{c}62-123 \\
28 \\
\text { oup: } 26(7\end{array}$ & $\begin{array}{c}62-133 \\
31 \\
\%)^{31}\end{array}$ & $\begin{array}{c}62-165 \\
33\end{array}$ \\
\hline $\begin{array}{l}\text { Group 2b } \\
\text { Depth range (m) } \\
\mathrm{N}^{\circ} \text { of stations } \\
\text { Stations always present }\end{array}$ & $\begin{array}{c}131-195 \\
21 \\
\text { roup: } 19\end{array}$ & $\begin{array}{c}131-275 \\
26 \\
7.9 \%)\end{array}$ & $\begin{array}{c}133-218 \\
28\end{array}$ & $\begin{array}{l}\text { Group } \mathbf{2 b} \\
\text { Depth range (m) } \\
\mathrm{N}^{\circ} \text { of stations } \\
\text { Stations always p }\end{array}$ & $\begin{array}{c}118-275 \\
27 \\
\text { oup: } 20(7\end{array}$ & $\begin{array}{l}118-249 \\
25\end{array}$ & $\begin{array}{c}131-218 \\
23\end{array}$ \\
\hline $\begin{array}{l}\text { Group } 3 \\
\text { Depth range (m) } \\
\mathrm{N}^{\circ} \text { of stations } \\
\text { Stations always present }\end{array}$ & $\begin{array}{c}202-413 \\
27 \\
\text { roup: } 27\end{array}$ & $\begin{array}{c}202-428 \\
27 \\
7.1 \%)\end{array}$ & $\begin{array}{c}202-505 \\
31\end{array}$ & $\begin{array}{l}\text { Group } 3 \\
\text { Depth range (m) } \\
\mathrm{N}^{\circ} \text { of stations } \\
\text { Stations always p }\end{array}$ & $\begin{array}{c}202-413 \\
24 \\
\text { oup: } 24(7\end{array}$ & $\begin{array}{l}202-434 \\
27 \\
\%)\end{array}$ & $\begin{array}{c}202-505 \\
31\end{array}$ \\
\hline $\begin{array}{l}\text { Group } 4 \\
\text { Depth range (m) } \\
\mathrm{N}^{\circ} \text { of stations } \\
\text { Stations always present }\end{array}$ & $\begin{array}{c}393-751 \\
31 \\
\text { roup: } 24\end{array}$ & $\begin{array}{c}454-751 \\
28 \\
7.4 \%)\end{array}$ & $\begin{array}{c}513-751 \\
24\end{array}$ & $\begin{array}{l}\text { Group } 4 \\
\text { Depth range (m) } \\
\mathrm{N}^{\circ} \text { of stations } \\
\text { Stations always p }\end{array}$ & $\begin{array}{c}393-751 \\
31 \\
\text { oup: } 24(7\end{array}$ & $\begin{array}{l}454-751 \\
28\end{array}$ & $\begin{array}{c}513-751 \\
24\end{array}$ \\
\hline
\end{tabular}



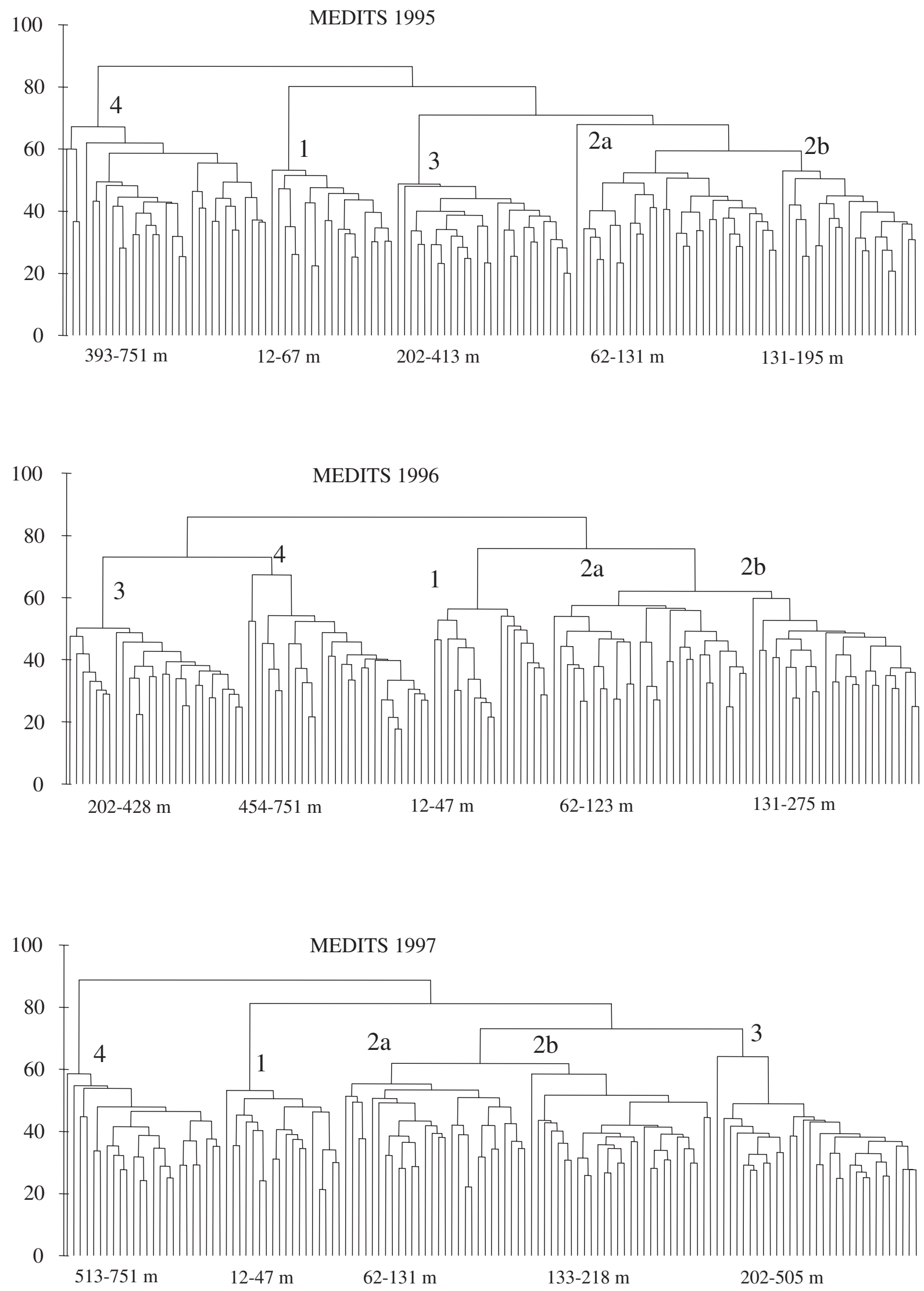

FIG. 2. - Dendrograms showing the groups of stations of each survey. Biomass data. Depth ranges within each major group are presented. 

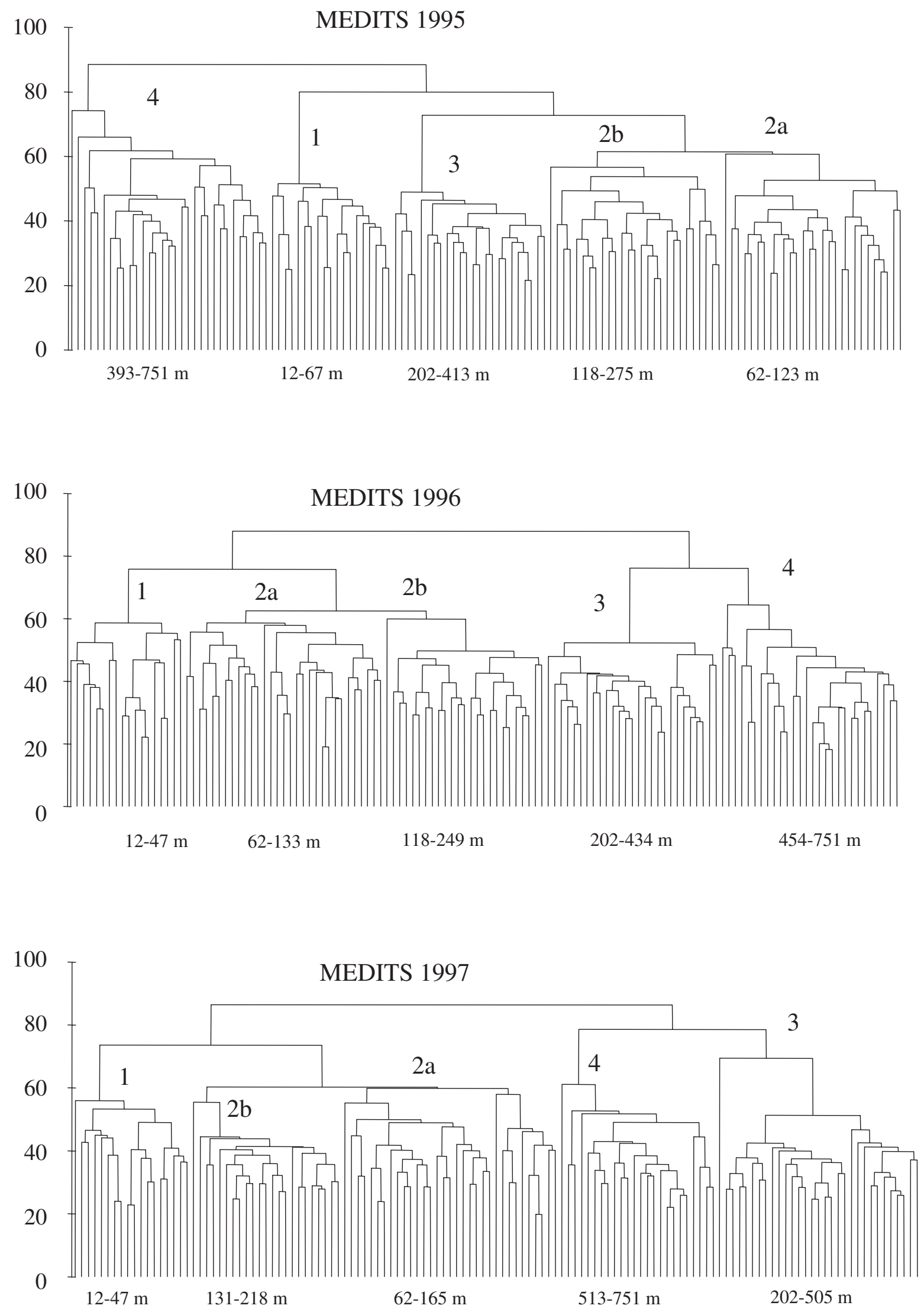

FIG. 3. - Dendrograms showing the groups of stations of each survey. Abundance data. Depth ranges within each major group are presented. 
TABLE 3. - The eight most important species, in terms of percentage contribution to the group similarity, listed for each group resulting from the cluster analysis. Biomass and abundance data.

\begin{tabular}{|c|c|c|c|c|c|}
\hline Group 1 & $\begin{array}{c}\text { Biomass } \\
\% \text { Sim }\end{array}$ & $\mathrm{kg} / \mathrm{km}^{2}$ & Species & $\begin{array}{r}\text { Abundance } \\
\% \text { Sim }\end{array}$ & $\mathrm{kg} / \mathrm{km}^{2}$ \\
\hline $\begin{array}{l}\text { D. annularis } \\
\text { Alloteuthis } \mathrm{spp} . \\
\text { P. erythrinus } \\
\text { A. laterna } \\
\text { T. mediterraneus } \\
\text { T. lucerna } \\
\text { L. vulgaris } \\
\text { M. barbatus }\end{array}$ & $\begin{array}{l}8.0 \\
7.7 \\
7.6 \\
6.9 \\
6.8 \\
5.3 \\
5.2 \\
4.7\end{array}$ & $\begin{array}{r}39.6 \\
4.4 \\
12.6 \\
1.6 \\
34.4 \\
2.1 \\
3.5 \\
28.6\end{array}$ & $\begin{array}{l}\text { T. mediterraneus } \\
\text { Alloteuthis spp. } \\
\text { D. annularis } \\
\text { S. flexuosa } \\
\text { P. erythrinus } \\
\text { A. laterna } \\
\text { M. barbatus } \\
\text { T. lucerna }\end{array}$ & $\begin{array}{r}10.8 \\
10.1 \\
9.3 \\
8.2 \\
6.6 \\
5.8 \\
5.4 \\
5.3\end{array}$ & $\begin{array}{r}1549.9 \\
1671.1 \\
1226.1 \\
1737.8 \\
422.7 \\
119.9 \\
909.7 \\
169.3\end{array}$ \\
\hline \multicolumn{6}{|l|}{ Group 2a } \\
\hline $\begin{array}{l}\text { M. merluccius } \\
\text { E. cirrhosa } \\
\text { Alloteuthis spp. } \\
\text { T. m. capelanus } \\
\text { T. trachurus } \\
\text { I. coindetii } \\
\text { A. laterna } \\
\text { L. cavillone }\end{array}$ & $\begin{array}{r}12.2 \\
6.8 \\
6.6 \\
6.3 \\
6.2 \\
5.3 \\
4.6 \\
3.5\end{array}$ & $\begin{array}{r}29.4 \\
13.5 \\
2.0 \\
10.5 \\
15.0 \\
12.2 \\
1.2 \\
3.3\end{array}$ & $\begin{array}{l}\text { M. merluccius } \\
\text { T. trachurus } \\
\text { T. m. capelanus } \\
\text { I. coindetii } \\
\text { Alloteuthis spp. } \\
\text { E. cirrhosa } \\
\text { L. cavillone } \\
\text { S. hepatus }\end{array}$ & $\begin{array}{r}12.2 \\
10.0 \\
6.8 \\
6.1 \\
5.9 \\
5.6 \\
4.0 \\
3.9\end{array}$ & $\begin{array}{r}2108.1 \\
2549.7 \\
1175.1 \\
156.7 \\
458.7 \\
141.0 \\
237.9 \\
294.9\end{array}$ \\
\hline \multicolumn{6}{|l|}{ Group 2b } \\
\hline $\begin{array}{l}\text { M. merluccius } \\
\text { E. cirrhosa } \\
\text { T. m. capelanus } \\
\text { I. coindetii } \\
\text { C. aper } \\
\text { T. trachurus } \\
\text { A. sphyraena } \\
\text { P. longirostris }\end{array}$ & $\begin{array}{r}10.3 \\
6.6 \\
6.6 \\
5.6 \\
5.1 \\
4.8 \\
4.8 \\
4.4\end{array}$ & $\begin{array}{r}80.8 \\
16.7 \\
14.7 \\
6.7 \\
6.8 \\
8.1 \\
2.2 \\
3.5\end{array}$ & $\begin{array}{l}\text { M. merluccius } \\
\text { T. m. capelanus } \\
\text { C. aper } \\
\text { I. coindetii } \\
\text { E. cirrhosa } \\
\text { A. sphyraena } \\
\text { T. trachurus } \\
\text { M. scolopax }\end{array}$ & $\begin{array}{r}13.5 \\
11.0 \\
6.0 \\
6.0 \\
5.5 \\
4.7 \\
4.3 \\
4.3\end{array}$ & $\begin{array}{r}8821.1 \\
1956.6 \\
1012.3 \\
219.9 \\
174.1 \\
509.1 \\
1305.9 \\
788.0\end{array}$ \\
\hline \multicolumn{6}{|l|}{ Group 3} \\
\hline $\begin{array}{l}\text { N. norvegicus } \\
\text { G. a. argenteus } \\
\text { E. cirrhosa } \\
\text { M. merluccius } \\
\text { P. blennoides } \\
\text { M. poutassou } \\
\text { S. oweniana } \\
\text { P. longirostris }\end{array}$ & $\begin{array}{l}7.4 \\
7.0 \\
6.4 \\
6.3 \\
5.9 \\
5.0 \\
4.5 \\
4.5\end{array}$ & $\begin{array}{r}11.4 \\
20.2 \\
19.9 \\
42.6 \\
7.6 \\
7.4 \\
2.6 \\
1.8\end{array}$ & $\begin{array}{l}\text { G. a. argenteus } \\
\text { S. oweniana } \\
\text { M. merluccius } \\
P . \text { blennoides } \\
\text { C. agassizi } \\
\text { N. norvegicus } \\
\text { P. longirostris } \\
\text { E. cirrhosa }\end{array}$ & $\begin{array}{r}12.4 \\
6.6 \\
5.7 \\
5.3 \\
5.2 \\
4.9 \\
4.4 \\
4.2\end{array}$ & $\begin{array}{r}8675.8 \\
831.3 \\
4493.4 \\
478.4 \\
854.7 \\
225.8 \\
166.4 \\
97.8\end{array}$ \\
\hline \multicolumn{6}{|l|}{ Group 4} \\
\hline $\begin{array}{l}\text { G. melastomus } \\
P . \text { blennoides } \\
\text { H. italicus } \\
\text { E. spinax } \\
\text { N. norvegicus } \\
\text { P. martia } \\
\text { N. sclerorhynchus } \\
\text { A. foliacea }\end{array}$ & $\begin{array}{r}11.9 \\
9.9 \\
6.7 \\
6.6 \\
6.2 \\
5.7 \\
5.7 \\
5.0\end{array}$ & $\begin{array}{r}51.1 \\
9.3 \\
1.8 \\
5.1 \\
7.3 \\
2.7 \\
6.0 \\
7.2\end{array}$ & $\begin{array}{l}\text { H. italicus } \\
\text { P. blennoides } \\
\text { P. martia } \\
\text { G. melastomus } \\
\text { N. norvegicus } \\
\text { L. crocodilus } \\
\text { N. sclerorhynchus } \\
\text { E. spinax }\end{array}$ & $\begin{array}{r}10.0 \\
9.3 \\
8.0 \\
7.6 \\
6.8 \\
5.6 \\
5.2 \\
4.7\end{array}$ & $\begin{array}{r}747.8 \\
738.1 \\
348.5 \\
316.1 \\
249.5 \\
282.8 \\
294.2 \\
93.7\end{array}$ \\
\hline
\end{tabular}

na and Mullus barbatus. These species presented high catch rates, especially in the case of T. mediterraneus and $D$. annularis, with, respectively, about 34 and $40 \mathrm{~kg}$ and 1,550 and 1,226 specimens per square kilometre.

In the shallower group of the continental shelf assemblage (group 2a), the aforesaid species were partially substituted by Merluccius merluccius, Eledone cirrhosa, Illex coindetii, Trachurus trachurus, and Trisopterus minutus capelanus. The biomass in this group was quite fairly distributed among these species, while the highest abundance values were registered for T. trachurus and M. merluccius.

Group $2 \mathrm{~b}$ was dominated by $M$. merluccius, both in terms of biomass and abundance. Other important species were E. cirrhosa, T. m. capelanus, Capros aper (especially in terms of abundance), Illex coindetii, Argentina sphyraena and T. trachurus.

Species characterising Group 3 were Gadiculus $a$. argenteus, M. merluccius, Phycis blennoides and 
Micromesistius poutassou, together with Nephrops norvegicus, E. cirrhosa and Sepietta oweniana (particularly important in terms of abundance). This group had the highest number of species in comparison to the others, essentially due to the fact that this depth range is a border line, being the upper bathymetric limit for some species and the lower for others.

The deepest group was characterised by Galeus melastomus with biomass index of about $51 \mathrm{~kg} / \mathrm{km}^{2}$, P. blennoides, Nezumia sclerorhynchus and Hymenocephalus italicus, Etmopterus spinax, Plesionika martia, Aristaeomorpha foliacea and $N$. norvegicus. In this last group most of the biomass caught corresponded to species or specimens without any commercial value.

\section{DISCUSSION}

One of the results of the "MEDITS" programme was its important contribution to the faunistic knowledge of the investigated areas. However, in most cases, the fact that one species was collected only in one sub-area does not always indicate a particular restricted geographic distribution. Generally, this aspect concerned species with a wide geographical distribution but only occasionally caught because of their low abundance and/or their reduced availability to the gear. In some cases, however, the presence of a species in only one sub-area is certainly due to its pattern of distribution. Particularly interesting is the case of the cephalopod Bathypolypus sponsalis, which was recorded in small numbers (less than 30 specimens per survey in the depth range $300-600 \mathrm{~m}$ ) only in sub-area 1 . This is in agreement with observations during ten years of national trawl surveys, which revealed its presence only north of the Island of Elba (Belcari, 1999). $B$. sponsalis is present in Atlantic and Mediterranean waters, but in the Italian Seas it shows an unhomogeneous distribution (Lumare, 1970, Würtz, 1979; Jereb et al., 1989; Mannini and Volpi, 1989; D’Onghia et al., 1993).

The spring-summer fish assemblages identified fit well in the general picture that the main biogeographic contours on continental shelves and slopes are strongly aligned with depth (Fager and Longhurst, 1968; Tyler et al., 1982; Overholtz and Tyler, 1985; Abelló et al., 1988; Biagi et al., 1989; Mahon and Smith, 1989; Sánchez et al., 1998; Demestre et al., 2000). Moreover, our results show a substantial agreement with previous works carried out in sub-sectors of the same area but with distinct sampling protocol and gear (Biagi et al., 1989; Abella and Serena, 1995 a and b).

Classificatory analysis of trawl data encompassing a period of three years indicated a high degree of spatial consistency in the clustering pattern of stations and in the species composition that characterise each cluster.

Persistence, i.e. the ability of an assemblage to maintain its species composition over time, is an important requirement for assemblage validity and, in addition, is an essential issue for management purposes (Pimm and Hyman, 1987; Tilman, 1996). The identification of such biological units, named by some authors APU (Assemblage Production Unit, Tyler et al., 1982), is basic to select a set of species that, due to their relative importance, might play an important role in the trophic relationships of the whole community. In a further step, functional trophic links among those species should be identified, so hence biological interactions (e.g. predator-prey relationships) would be incorporated in fishery management, based on a Multispecies Virtual Population Analysis (MSVPA, Helgason and Gislason, 1979; Pope, 1979; Daan and Sissenwine, 1991).

The present work should be seen as a useful data source to identify the appropriate spatial scale for a multispecies fishery study. Thus, this definition of broad geographic areas, characterised by a relatively homogeneous and persistent biological composition, represents the first step to deal with before trying to incorporate an ecosystem approach into practical fishery management advice.

A natural evolution of the present work should analyse assemblage structure by examining size spectrum composition. Moreover, theoretical studies indicate that for exploited systems, multiple equilibrium states of community structure are possible and, therefore, changes in structure are unlikely to be reversible. There is thus an important need to monitor the structure of faunistic assemblages over the time.

\section{ACKNOWLEDGEMENTS}

We wish to thank Dr. Mario Mori for his significant help in the identification of crustaceans. We also thank the crew of the fishing vessel "Francesco Padre" and all the scientists that, in different ways, contributed to the success of the surveys. 


\section{REFERENCES}

Abella, A. and F. Serena. - 1995a. Definition of the groundifsh assemblages caught off the Tuscanian coasts for fisheries purposes. Rapp. Comm. int. Mer Médit., 43: 235.

Abella, A. and F. Serena. - 1995b. Definizione di assemblaggi demersali nell'Alto Tirreno. Biol. Mar. Medit., 2(2): 451-453.

Abelló, P., F.J. Valladares and A. Castellón. - 1988. Analysis of the structure of decapod crustacean assemblages off the Catalan coast (north-west Mediterranean). Mar. Biol., 98: 39-49.

Belcari, P. - 1999. Bathypolipus sponsalis. In: G. Relini, J. Bertand and A. Zamboni (eds.), Synthesis of the knowledge on bottom fishery resources in central Mediterranean (Italy and Corsica). Biol. Mar. Medit., 6 (suppl): 733-736.

Bertrand, J.A., L. Gil de Sola, C. Papaconstantinou, G. Relini and A. Souplet. - 2000. An international bottom trawl survey in the Mediterranean: the MEDITS programme. In: J.A. Bertrand and G. Relini (eds.), Demersal resources in the Mediterranean, Proceedings of the Symposium held in Pisa, 18-21 March 1998, Actes de Colloques 26, pp. 76-93. IFREMER, Plouzané.

Bertrand, J., L. Gil de Sola, C. Papaconstantinou, G. Relini and A. Souplet. - 2002. The general specifications of the MEDITS surveys. Sci. Mar., 66 (Suppl. 2): 9-17.

Biagi, F., S. De Ranieri, M. Mori, P. Sartor and M. Sbrana. - 1989. Preliminary analysis of demersal fish assemblages in the northern Tyrrhenian Sea. Nova Thalassia, 10 (suppl. 1): 391-398.

Brugge, W.J. and M.J. Holden. - 1991. Multispecies management: a manager's point of view. ICES Mar. Sci. Symp., 193: 353-358.

Caddy, J.F. and G.D. Sharp. - 1986. An ecological framework for marine fishery investigations. FAO Fish. Tech. Pap., 283: 1-152.

Clarke, K.R. and R.M. Warwick. - 1994. Change in marine communities: an approach to statistical analysis and interpretation. Natural Environmental Research Council - Plymouth Marine Laboratory, U.K.

Clifford, H.T. and W. Stephenson. - 1975. An introduction to numerical classification. Academic Press, New York.

Daan, N. and M.P. Sissenwine. - 1991. Multispecies models relevant to management of living resources. ICES MSS, 193: 1-358.

Demestre, M., P. Sánchez and P. Abelló. - 2000. Demersal fish assemblages and habitat characteristics on the continental shelf and upper slope of the north-western Mediterranean. J. mar. biol. Ass. U.K., 80: 981-988.

D’Onghia, G., A. Tursi, A. Matarrese and P. Panetta. - 1993. Some aspects of the biology of Bathypolypus sponsalis (Mollusca, Cephalopoda) in the North Aegean Sea (Eastern Mediterranean Sea). Vie Milieu, 43 (2-3): 161-164.

Fager, E.W. and A.R. Longhurst. - 1968. Recurrent group analysis of species assemblages of demersal fish in the Gulf of Guinea. J. Fish. Res. Bd., 25(7): 1405-1421.

Fredj, G., D. Bellan-Santini and M. Meinardi. - 1992. État des connaissances sur la faune méditerranéenne. Bull. Inst. Océanogr. Monaco, special issue 9: 133-145.

Helgason, T. and H. Gislason. - 1979. VPA-analysis with species interaction due to predation. ICES C.M., 1979/G/52: 1-10.

Jereb. P., R. Baino, S. Ragonese and P. Mannini. - 1989. Bathypolypus sponsalis (P. et H. Fisher, 1892). Nova Thalassia, 10 (suppl. 1): 513

Lumare. F. - 1970. Nota sulla distribuzione di alcuni Cefalopodi del
Mar Tirreno. Boll. Pesca Piscic. Idrobiol., 25(2): 313-344.

Mahon, R. and R.W. Smith. - 1989. Demersal fish assemblages on the Scotian shelf, north-west Atlantic: spatial distribution and persistence. Can. J. Aquat. Fish. Sci., 46: 134-152.

Mangold, K and S.V. Boletzky. - 1987. Cephalopodes. In: W. Fisher, M.L. Bauchot and M. Schneider (eds.), Fiches FAO d'identification des espèces pour les besoins de la pêche. (Revision 1). Mediterranée et Mer Noir. Zone de pêche 37. Volume I. Invertebrés, pp. 633-714. Rome, FAO.

Mannini, P. and C. Volpi. - 1989. Nota sulla presenza e distribuzione di alcuni Cefalopodi del Tirreno settentrionale. Oebalia, 15(2): 693-701.

Maurin, C. - 1962. Étude des fonds chalutables de la Méditerranée occidentale (écologie et pêche). Résultats des campagnes des navires océanographiques "Président Théodore Tissier" 1957 à 1960 et "Thalassa" 1960 et 1961. Rev. Trav. Inst. Peches Marit., Paris, 26(2): 163-218.

Overholtz, W.J. and A.V. Tyler. - 1985. Long-term responses of the demersal fish assemblages of George's Bank. Fish. Bull., 83: 507-520.

Pimm, L.S. and J.B. Hyman. - 1987. Ecological stability in the context of multispecies fisheries. Can. J. Fish. Aquat. Sci., 44: 84-94.

Pope, J.G. - 1979. Stock assessment in multispecies fisheries, with special reference to the trawl fisheries in the Gulf of Thailand. SCS/DEV/79/19. South China Sea Fisheries Development and Coordinating Programme, Manila, 106 pp.

Sánchez, P., P. Belcari and P. Sartor. - 1998. Composition and spatial distribution of cephalopods in two north-western Mediterranean areas. S. Afr. J. mar. Sci., 20: 17-24.

Sneath, P.H.A. and R.R. Sokal. - 1973. Numerical taxonomy. The principles and practice of numerical classification. W.H. Freeman and Company, San Francisco.

Souplet, A. - 1996. Calculation of abundance indices and length frequencies in the MEDITS survey. In: J.A. Bertrand et al. (eds.), Campagne internationale de chalutage demersal en Mediterranee. Campagne 1995. EU Final Report, Vol. III.

Tilman, D. - 1996. Biodiversity: population versus ecosystem stability. Ecology 77: 350-363.

Tortonese, E. - 1987. Pesci nel Mediterraneo. Recenti studi intorno alla sistematica e distribuzione. Quad. Istit. Idrob. Acquac. "G. Brunelli", Roma: 1-111.

Tyler, A.V. - 1988. Biological basis for management of groundfish resources in the west coast of Canada. In: W.S. Wooster (ed.), Fishery Science and management: objectives and limitations, pp. 217-232. Springer-Verlag, Berlin.

Tyler, A.V., W.L. Gabriel and W.J Overholtz. - 1982. Adaptive management based on structure of fish assemblage of northern continental shelves. Can. Spec. Publ. Fish., 59: 149-156.

Whitehead, P.J.P., M.L. Bauchot, J.C. Hureau, J. Nielsen and E. Tortonese. - 1984. Fishes of the north-eastern Atlantic and Mediterranean (FNAM), Vol. 1. UNESCO, Paris.

Whitehead, P.J.P., M.L. Bauchot, J.C. Hureau, J. Nielsen and E. Tortonese. - 1986. Fishes of the north-eastern Atlantic and Mediterranean (FNAM). Vols. 2-3. UNESCO, Paris.

Würtz, M. - 1979. I cefalopodi raccolti in mar Ligure durante la campagna di pesca batiale 1977/1978. Atti Soc. Tosc. Sci. Nat. Mem. Ser. B, 86: 374-377.

Zariquiey Álvarez, R. - 1968. Crustáceos decápodos ibéricos. Inv. Pesq., 32: 1-510. 